\title{
Skin cancer awareness and sunscreen use among outpatients of a South African hospital: need for vigorous public education
}

\author{
NC Dlova ${ }^{a *}$, R Gathers $^{b}$, J Tsoka-Gwegwenic and RJ Hift ${ }^{d}$ \\ aDiscipline of Dermatology, Nelson R Mandela School of Medicine, University of KwaZulu-Natal, Durban, South Africa \\ ${ }^{b}$ Department of Dermatology, Multicultural Dermatology Center, Henry Ford Hospital, Detroit, Michigan \\ 'Department of Public Health Medicine, School of Nursing and Public Health, University of KwaZulu-Natal, Durban, South Africa \\ ${ }^{d}$ School of Clinical Medicine, University of KwaZulu-Natal, Durban, South Africa \\ *Corresponding author, email:dlovan@ukzn.ac.za
}

\begin{abstract}
Background: Melanoma incidence among white South Africans is increasing. Among black individuals, melanoma is associated with advanced stage at presentation and significant mortality.

Objectives: A study was undertaken to assess the perception of skin cancer risk, knowledge of skin cancer, and understanding of the importance and use of sunscreens among South Africans.

Methods: A cross-sectional survey was conducted in general outpatients over four months at a large central hospital in Durban, South Africa.

Results: Only half the white respondents reported regular use of a sunscreen. Among black and Indian respondents, the number was substantially lower. Less than $20 \%$ of white respondents had ever checked their skin for suspicious moles. Most black patients were not aware that they are at risk of skin cancer, and only $10 \%$ were aware of the risk of developing skin cancers on acral sites and nails.

Conclusions: There is a worrying lack of knowledge about skin cancer and sun protection behaviours among all South Africans. Given the increase in melanoma incidence and racial disparities in survival rates, it is imperative to target each population with effective, culturally sensitive educational programmes.
\end{abstract}

Keywords: education, melanoma, prevention, screening, skin cancer, South Africa, sunscreen

\section{Introduction}

Malignant melanoma is a potentially devastating disease. The strong association of ultraviolet exposure with skin cancer makes prevention through measures such as sun-avoidance and protective clothing essential. Early detection of melanoma is critical in order to allow its diagnosis before metastasis, whilst it is still amenable to local excision. Routine screening examination, both self-examination and examination by health practitioners, is important. ${ }^{1-3}$

Despite efforts to encourage preventive measures, ${ }^{4}$ melanoma incidence has increased over the last three decades. The agestandardised melanoma incidence rate among the white population in Cape Town was reported as 24.4 per 100000 in $1998,{ }^{5}$ but was found to be 33.5 and 36.9 per 100000 for women and men respectively in 2008. ${ }^{6}$ That melanoma incidence continues to rise in South Africa possibly suggesting a substantial knowledge deficit concerning the condition and the steps necessary to prevent it.

Given the protective pigmentation, the incidence is substantially lower in black Africans. Incidence rates between 0.9 and 1.2 per 100000 among black South Africans have been reported. ${ }^{7,8}$ However, the advantage of this is offset by a tendency to late presentation. Some $70 \%$ of melanomas in black Africans are reported to be on the lower limbs, with $90 \%$ of those being below the ankle, with acral lentiginous melanoma being the most common subtype., ${ }^{3,9}$ The plantar, subungual and mucosal surfaces are common sites for melanoma in black South African patients. ${ }^{3,10}$ Whereas $71-74 \%$ of melanomas in the white South African population have been reported to have Breslow thickness $<1.5 \mathrm{~mm},{ }^{5}$ black patients present with significantly higher Breslow thickness depths. ${ }^{11}$ Survival rates of plantar melanoma in black patients are very low-only $25 \%$ of a sample of 40 black African patients with plantar melanoma survived five years. ${ }^{11}$ The South African melanoma data parallel US SEER (Surveillance, Epidemiology, and End Results Program) data in terms of the race-correlated differences in both incidence and thickness depth at presentation. ${ }^{4,12,13}$ In the USA AfricanAmericans are more likely to present with advanced disease and to have a worse prognosis than white individuals. ${ }^{14}$

Durban is a coastal city situated at latitude $29^{\circ} \mathrm{S}, 5^{\circ}$ closer to the equator than Sydney, Australia. The ambient erythema ultraviolet radiation (EUV) dose is $25-35$ minimal erythema dose (MED) units per day in summer, ${ }^{15}$ depicting high levels of UV exposure. Given the substantial ethnic differences in melanoma incidence and outcome, we hypothesised that this would be accompanied by corresponding differences in the level of understanding of skin cancer and its prevention between the groups at low risk and high risk of disease. We therefore undertook a study to determine the perception of skin cancer risk, skin cancer appearance, and general knowledge regarding sun protection among our multi-ethnic population. The objectives of our study were to assess the understanding of the presentation of melanoma and its risks, the importance of sun protection and early recognition in prevention and the effects of current attributes associated with ethnicity or race and other factors involved in determining understanding and behaviour. 


\section{Materials and methods}

A cross-sectional survey was conducted in the outpatient department of Albert Luthuli Central Hospital, a major tertiary referral centre in Durban, KwaZulu-Natal Province. Patients reporting for registration in the hospital's common reception area for outpatients between September 1 and December 31, 2012 were approached. South African citizens aged between 18 and 90 years attending outpatient clinics other than the dermatology clinic were invited to participate; such clinics therefore spanned the whole breadth of the service profile offered by this hospital.

We designed a structured questionnaire, which elicited answers on a range of topics (see Results, and Table 1). The first seven questions interrogated knowledge of sun protection, skin cancer and its recognition, then an understanding of the relationship between sun exposure and sun damage, and lastly evidence of appropriate behaviours for the prevention or early recognition of skin cancer. The questionnaire was initially administered to 20 subjects for validation, following which a trained study assistant who was proficient in both English and isiZulu, the locally used African language, administered it to the study subjects. Subjects were not subject to clinical examination.

\section{Data analysis}

Data were entered into a Microsoft Excel ${ }^{\circledR}$ spreadsheet (Microsoft Corporation, Redmond WA, USA) and analysed with MedCalc ${ }^{\varpi}$ Statistical Software version 12.7.5 (MedCalc Software BVBA, Ostend, Belgium). Data were summarised in contingency tables and analysed for significance using a chi-square test or Fisher's exact test as appropriate. Ordinal data were compared between groups by Student's $t$-test except where otherwise reported.

\section{Results}

One thousand subjects were invited to participate of whom 963 participants provided consent and were interviewed. Seventeen mixed-race patients $(1.7 \%)$ were excluded from the analysis. Demographic data are summarised in Figure 1. Thirty-four of the subjects reported that they had previously had skin cancer. Their level of knowledge on the first 10 knowledge questions was significantly higher than those who had not had previous experience of skin cancer (median score 7 versus median score $3.5, p<0.0001$ ), though their behaviour did not differ (median score 1 for both groups, $p=0.52$ ).

Results are summarised in Table 1. The median number of appropriate answers on the 10 knowledge questions was one for black African respondents (IQR $0-4)$, four for Indian respondents (IQR 1-6) and five for white respondents (IQR 3-6) $(p<0.0001)$. Ranked on the 10 knowledge topics, the median score for females was 5 (IQR 1-6), and for males 2 (IQR $0-5)(p<0.0001)$. Race, gender, educational achievement, younger age and active employment were significantly associated with higher questionnaire scores on logistic regression. Females scored significantly better than males on every question. Two-way ANOVA showed significant interactions between race and education, and race and age. Race remained the strongest predictor of a high score, even after controlling for education, age and employment.

White respondents were significantly more likely to practise appropriate skin-protective behaviours (median 2) than black African or Indian respondents (median $1, p<0.0001$ ). The number of correct answers on the knowledge questions correlated significantly with the likelihood of practising skin-protective behaviour $(r=0.52, p<0.0001)$. Eighty respondents $(8.4 \%)$ reported ever having had a conversation with a doctor about the prevention and recognition of skin cancer. Results are summarised in Figure 2.

Table 1: Demographic data and awareness of specific aspects of skin cancer, sun protection and prevention of skin damage, and appropriate behaviours reported by 963 respondents

\begin{tabular}{|c|c|c|c|c|c|}
\hline Question & Black African $(n=373)$ & Indian $(n=308)$ & White $(n=282)$ & All $(n=963)$ & $p$-value \\
\hline \multicolumn{6}{|l|}{ Knowledge: } \\
\hline $\begin{array}{l}\text { Awareness that black populations (including African and } \\
\text { Indian people) are at risk of skin cancer }\end{array}$ & $173(46.4 \%)$ & $225(73.1 \%)$ & $240(85.1 \%)$ & $638(66.3 \%)$ & $<0.0001$ \\
\hline $\begin{array}{l}\text { Awareness that regular use of sunscreen may prevent skin } \\
\text { cancer }\end{array}$ & $113(30.3 \%)$ & $178(57.8 \%)$ & $229(81.2 \%)$ & $520(54.0 \%)$ & $<0.0001$ \\
\hline Understanding of the concept of sun protection factor (SPF) & $110(29.5 \%)$ & $165(53.6 \%)$ & $239(84.8 \%)$ & $514(53.4 \%)$ & $<0.0001$ \\
\hline Awareness that sunburn is a form of skin damage & $43(11.8 \%)$ & $89(29.1 \%)$ & $164(59.0 \%)$ & $296(31.2 \%)$ & $<0.0001$ \\
\hline Awareness of the need for regular sunscreen use & $59(15.8 \%)$ & $76(24.7 \%)$ & $60(21.3 \%)$ & $195(50.2 \%)$ & 0.015 \\
\hline Sufficient knowledge to recognise a suspicious skin lesion & $24(6.4 \%)$ & $37(12.0 \%)$ & $51(18.1 \%)$ & $112(11.6 \%)$ & $<0.0001$ \\
\hline $\begin{array}{l}\text { Awareness that melanoma may present on palms, soles and } \\
\text { nails }\end{array}$ & $37(9.9 \%)$ & $39(12.7 \%)$ & $21(7.4 \%)$ & $97(10.1 \%)$ & 0.1 \\
\hline \multicolumn{6}{|l|}{ Cosmesis: } \\
\hline Awareness that sunscreen promotes an even skin tone & $89(23.9 \%)$ & $137(44.5 \%)$ & $157(55.7 \%)$ & $383(39.8 \%)$ & $<0.0001$ \\
\hline Awareness that sunscreen can prevent wrinkling & $73(19.6 \%)$ & $101(32.8 \%)$ & $162(57.4 \%)$ & $336(34.9 \%)$ & $<0.0001$ \\
\hline Awareness that sunscreen can prevent darkening of the skin & $85(22.8 \%)$ & $147(47.7 \%)$ & $64(22.7 \%)$ & $296(30.7 \%)$ & $<0.0001$ \\
\hline \multicolumn{6}{|l|}{ Behaviour: } \\
\hline Avoidance of sunbathing & $332(89.0 \%)$ & $201(65.3 \%)$ & $247(87.6 \%)$ & $780(81.0 \%)$ & $<0.0001$ \\
\hline Correct use of sunscreen & $52(13.9 \%)$ & $68(22.1 \%)$ & $145(51.4 \%)$ & $265(27.5 \%)$ & $<0.0001$ \\
\hline Skin self-examination & $17(4.6 \%)$ & $43(14.0 \%)$ & $51(18.1 \%)$ & $111(11.5 \%)$ & $<0.0001$ \\
\hline Skin examination by a doctor & $15(4.0 \%)$ & $23(7.5 \%)$ & $24(8.6 \%)$ & $62(6.5 \%)$ & 0.08 \\
\hline
\end{tabular}




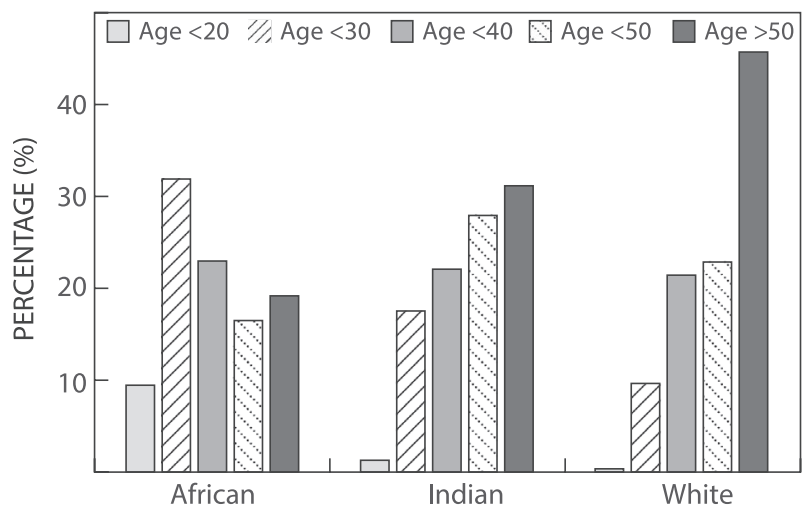

Figure 1: Age distribution of the sample group by ethnicity. [Age distribution of respondents, categorised by race].

\section{Discussion}

South Africa has a high incidence of skin cancer. Given that skin cancer is preventable through behavioural modification such as sun avoidance and the use of sunscreens, and that early detection translates directly into better outcomes, it is essential that the population understands these factors. It is concerning that the general level of knowledge among our respondents was poor.

We found significant differences in the levels of knowledge in the three ethnic groups. Though the groups themselves were somewhat heterogeneous, with differences in age, gender, educational level and employment status, we found a correlation between race and understanding, even after controlling for factors such as gender, education and age, which were independently associated with understanding. We believe that a lack of awareness of melanoma risk amongst dark-skinned people may be a key factor. The white population by contrast is continually exposed to articles in the mainstream press, targeted advertisements for sunscreen and keen parenting resources that might result in greater knowledge and awareness of skin cancer and the importance of sun protection.

Although black Africans are at lower risk of melanoma than lighter-skinned groups, the risk is not negligible and may be reduced by limiting sun exposure or using sunscreens. It is alarming that only $46 \%$ of the black African respondents were aware of their risk of skin cancer, and 30\% that they should use sunscreen. The risk is further increased by their lack of knowledge that melanoma may present on the palms, soles and nails, sites typical of the lentiginous acral melanoma most commonly affecting black people., ${ }^{3,10}$ Furthermore, only $7 \%$ felt that they would recognise a suspicious skin lesion. Aside from melanoma, basal cell carcinoma (BCC) has been reported to represent 12 $35 \%$ of skin cancers in African-Americans, and $2-8 \%$ of skin cancers in black Africans. ${ }^{13}$ Further, the majority of BCCs in black African and Indian people occur in sun-exposed skin suggesting that sun protection should be practised, regardless of pigment. ${ }^{13}$ Perceived skin cancer risk may correlate with risk-averse behaviours. Likewise, a lack of perceived risk may be a barrier to risk reduction. ${ }^{16,17}$ The incidence of melanoma is strongly influenced by ethnicity. Most public education programs for melanoma are directed towards white people. ${ }^{14} \mathrm{~A}$ consequence of this may be that ethnic groups with darker skin, including black and Indian people, may not be reached by such programmes, despite being at some risk of melanoma. An understanding of the relationship between sun exposure and risk of skin cancer is fundamental to prevention.

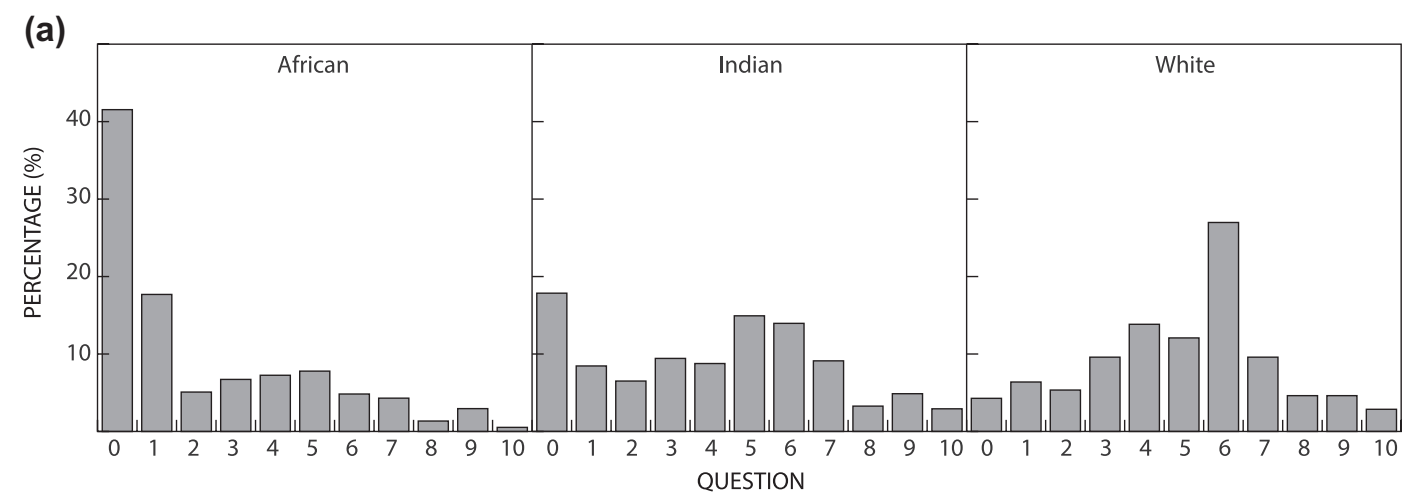

(b)

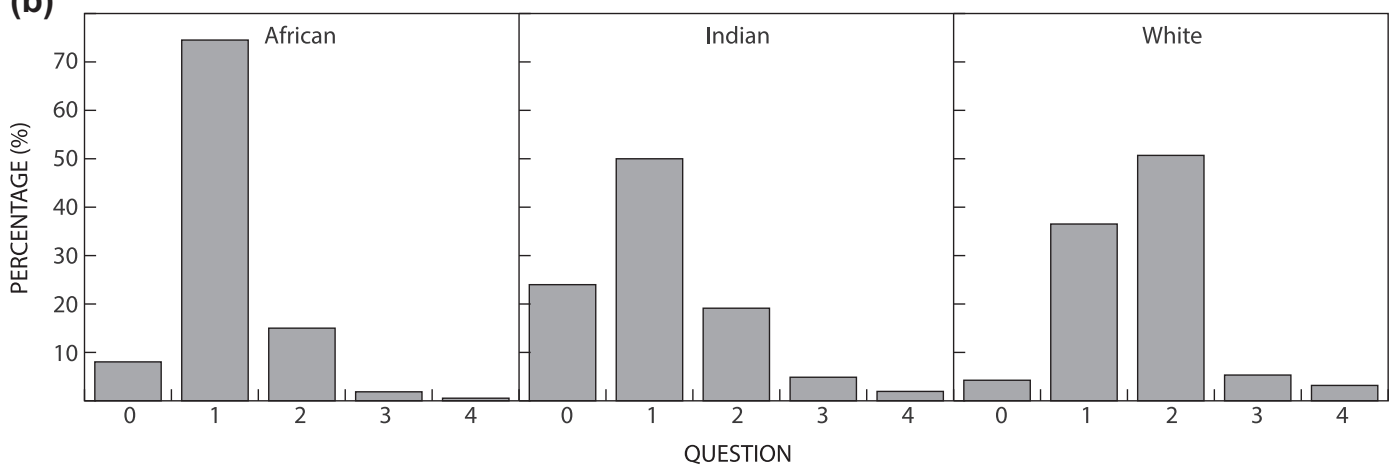

Figure 2: Distribution of scores on the knowledge items (top panel) and on behaviour items (lower panel). [Top panel: distribution plot of the number of areas appropriately understood (see Table 1) on a series of 10 questions testing knowledge of sun protection and skin cancer prevention and recognition. Bottom panel: distribution plot of the number of appropriate behaviours reported by the respondents. The behaviours are sun avoidance, regular use of sunscreen, skin self-examination and skin examination by a doctor]. 
The benefits of sunscreen are not restricted to cancer prevention. Sunscreen may reduce wrinkling and photo damage, resulting in more even pigmentation. With age, darker skin types are more likely to demonstrate skin mottling and uneven skin pigmentation..$^{18,19}$ Our results suggest a general lack of understanding of the link between light skin, propensity for sunburn, risk of melanoma and of the role of sun protection in ameliorating this risk. Though most white respondents are appropriately aware that regular use of sunscreen may prevent skin cancer and understand the concept of sun-protection factor, it is concerning that $41 \%$ have not understood that sunburn is a form of skin injury, and only $21 \%$ expressed an awareness of the need for regular use of sunscreen.

Understanding the link between sun exposure and skin damage, the protective effects of sunscreens against photo ageing, and the concept of the sun protection factor (SPF) was significantly poorer among black African respondents: just $11 \%$ reported frequent recreational sun exposure. This is likely to be culturally mediated rather than for reasons of health, a fairer (i.e. nontanned) skin being pervasively and historically regarded as more attractive in females of both Asian and some African societies, particularly South Africa. ${ }^{20-26}$ It is known that there is a strong cultural propensity to prefer light skin rather than dark skin amongst our black African and Indian population, and that skinbleaching practices are common in this population. Prevalence rates of skin bleaching in sub-Saharan Africa have been reported as between $26 \%$ and $67 \%$, despite an array of potential adverse side effects from the practice. ${ }^{27}$ Though the desire for a lighter complexion might be expected to be a powerful driver for regular use of sunscreen, our respondents showed little understanding of sunscreen use in terms of SPF, the need for regular use in the black African population and its role in cosmesis. Female respondents were twice as likely to answer positively on these questions as males ( $p<0.0001)$, a wider margin than was shown for the knowledge questions in general.

The Indian population was particularly marked for its preference for recreational sun exposure, claimed by nearly $40 \%$. This was coupled with an intermediate but generally suboptimal level of knowledge of sun and skin cancer and its presentation, even though they share the same skin types as the black African population. Paradoxically, it appears that our dark-skinned population are more likely to use bleaching products to lighten skin tone, often with adverse consequences, than to use sunscreen to reduce the darkening effects of the sun in the first place. Indeed, increased sunscreen usage and education regarding sun avoidance among black African and Indian people might substantially reduce the perceived need for skin-bleaching products.

Of concern is the lack of ability to recognise suspicious skin lesions and the very low percentage of subjects who practise skin self-examination. Although the white respondents practise significantly more skin-friendly behaviours, few subjects used sunscreens regularly, inspected their own skins and underwent regular medical skin examinations (see Figure 2).

On logistic regression, the likelihood of complying with two or more of these practices correlated significantly with female gender, white race, post-primary education, employment and particularly with the level of understanding shown by the 10 knowledge questions. Few respondents had had their skin examined by a doctor. The examination was generally performed by a dermatologist, suggesting that family practitioners and other health-care professionals are neglecting this important aspect of the physical examination. The low percentage of respondents who had ever discussed skin cancer with a doctor supports this. This may reflect deficiencies in medical training, which lacks emphasis on skin cancer screening.

\section{Conclusions}

Our results indicate a serious deficit in knowledge of sun protection and the prevention and recognition of skin cancer, as well as a lack of risk-averse behaviours in our population. Those population groups with a darker skin in particular are unaware of the risks posed by sun damage and skin cancer. The South African population requires intensive education regarding the benefits of using sunscreen not only to prevent skin cancer but also to obviate skin mottling and uneven skin tone, a characteristic feature in this population. This in turn may lessen some of the dependency of our black African population on skin-lightening preparations. Yet both the knowledge and the protective behaviours of the white population are still grossly suboptimal, despite the very high incidence of skin cancer in this group.

The high incidence of melanoma in white patients and the disproportionate mortality burden of melanoma in black African patients mandate public health and screening initiatives. There is a need to convey the message that hands, feet and nails are vulnerable in the black population, and should be inspected regularly. The results indicate the need for targeted and culturally inclusive programmes to increase the rate of early detection, decrease melanoma incidence and improve general skin health. Physicians and health-care providers should take a lead in instituting early detection and screening measures, and in educating the public on the need for these.

Ethical approval - Received from the University of KwaZulu-Natal Biomedical Research Ethics Committee (BE 180/11). We studied the three largest ethnic groups in Durban: indigenous African, Indian (a large population founded by South-Asian immigrants in the nineteenth century), both of whom are of Fitzpatrick skin types 4-6, and white (Fitzpatrick types 1-4). A power analysis suggested that 300 subjects from each ethnic group were required to demonstrate significant differences in responses. All subjects provided written informed consent.

Acknowledgements - The authors would like to thank their colleagues in the Department of Dermatology, Ms Phakama Jika, Ms Lungie Shabalala and Mrs T. Yeni of Durban for data collection and administrative support.

Funding - Prof. Ncoza Dlova is supported by a Discovery Foundation Academic fellowship award, a Dermatological Society of South Africa research grant, the University of KwaZuluNatal (UKZN) College of Health Sciences Strategic Research Fund, the UKZN Competitive Research Fund, a National Research Foundation Indigenous Knowledge Systems grant, a Medical Education Partnership Initiative (MEPI) grant and is a Fellow of the UKZN Leadership and Equity Advancement Programme.

Disclosure statement - No potential conflict of interest was reported by the authors.

\section{References}

1. Stryker JE, Solky BA, Emmons KM. A content analysis of news coverage of skin cancer prevention and detection, 1979 to 2003. Arch Dermatol. 2005;141(4): 491-96.

2. Aitken JF, Elwood M, Baade PD, et al. Clinical whole-body skin examination reduces the incidence of thick melanomas. Int J Cancer 2010;126(2): 450-58. https://doi.org/10.1002/ijc.v126:2 
3. Krige JEJ. Melanoma in black South Africans. S Afr J Surg. 2010;48(3): 74-75.

4. Little EG, Eide MJ. Update on the current state of melanoma incidence. Dermatol Clin. 2012;30(3): 355-61. https://doi.org/10.1016/j. det.2012.04.001

5. Saxe N, Hoffman M, Krige JE, et al. Malignant melanoma in Cape Town, South Africa. Br J Dermatol. 1998;138(6): 998-1002. https://doi. org/10.1046/j.1365-2133.1998.02266.x

6. Jessop S, Stubbings $H$, Sayed R, et al. Regional clinical registry data show increased incidence of cutaneous melanoma in Cape Town. $S$ Afr Med J. 2008;98(3): 197-99.

7. Isaacson C, Spector I. Malignant melanomas in the Eur-African-Malay population of South Africa. Am J Dermatopathol. 1987;9(2): 109-10. https://doi.org/10.1097/00000372-198704000-00004

8. Giraud RM, Rippey E, Rippey JJ. Malignant melanoma of the skin in Black Africans. S Afr Med J. 1975;49(16): 665-68.

9. Hudson DA, Krige JE. Melanoma in black South Africans. J Am Coll Surg. 1995;180(1): 65-71.

10. Lodder JV, Simson W, Becker PJ. Malignant melanoma of the skin in black South Africans: A 15-year experience. S Afr J Surg. 2010;48: 76-79.

11. Hudson DA, Krige JE. Plantar melanoma in black South Africans. Br J Surg. 1993;80(8): 992-94. https://doi.org/10.1002/(ISSN)1365-2168

12. Hu S, Parmet $Y$, Allen $G$, et al. Disparity in melanoma: a trend analysis of melanoma incidence and stage at diagnosis among whites, Hispanics, and blacks in Florida. Arch Dermatol. 2009;145(12): 136974.

13. Gloster HM Jr, Neal K. Skin cancer in skin of color. J Am Acad Dermatol. 2006;55(5): 741-60. https://doi.org/10.1016/j.jaad.2005.08.063

14. Byrd KM, Wilson DC, Hoyler SS, et al. Advanced presentation of melanoma in African Americans. J Am Acad Dermatol. 2004;50(1): 21-4. https://doi.org/10.1016/S0190-9622(03)02091-7

15. Guy CY, Diab RD. A health risk assessment of ultraviolet radiation in Durban. S Afr Geogr J. 2002;84(2): 208-13. https://doi.org/10.1080/03 736245.2002.9713772
16. Buster KJ, You Z, Fouad M, et al. Skin cancer risk perceptions: a comparison across ethnicity, age, education, gender, and income. J Am Acad Dermatol. 2012;66(5): 771-79. https://doi.org/10.1016/j. jaad.2011.05.021

17. Vernon SW. Perception and risk communication for cancer screening behaviors: a review. J Natl Cancer Inst. 1999;91(18): 101-19. https:// doi.org/10.1093/oxfordjournals.jncimonographs.a024184

18. Alexis AF, Rossi A. Photoaging in skin of color. Cosmet Dermatol. 2011;24: 367-70.

19. Rawlings AV. Ethnic skin types: are there differences in skin structure and function? Int J Cosmet Sci. 2006;28(2): 79-93. https://doi. org/10.1111/ics.2006.28.issue-2

20. Li EP, Min HJ, Belk RW. Skin lightening and beauty in four Asian cultures. NA-Adv Consumer Res. 2008;35.

21. Dlova N, Hamed S, Tsoka-Gwegweni J, et al. Skin lightening practices: an epidemiological study of South African women of African and Indian ancestries. $\mathrm{Br} J$ Dermatol. 2015;173(S2): 2-9. https://doi. org/10.1111/bjd.13556

22. Naidoo L, Khoza N, Dlova NC. A fairer face, a fairer tomorrow? A review of skin lighteners. Cosmetics 2016;3(3): 33. https://doi.org/10.3390/ cosmetics 3030033

23. Thomas LM. Skin lighteners in South Africa: Transnational entanglements and technologies of the self. In: Shades of difference: Why skin color matters. 2009. p. 188-210.

24. Ntambwe M. Mirror mirror on the wall, who is the fairest of them all. Sci Africa. 2004.

25. Hunter ML. Buying racial capital: Skin-bleaching and cosmetic surgery in a globalized world. JPAS. 2011;4(4): 142-64.

26. Blay YA. Skin bleaching and global white supremacy: By way of introduction. JPAS. 2011;4(4): 4-46.

27. Dadzie OE, Petit A. Skin bleaching: highlighting the misuse of cutaneous depigmenting agents. J Eur Acad Dermatol Venereol. 2009;23(7): 741-50. https://doi.org/10.1111/jdv.2009.23.issue-7

Received: 14-07-2017 Accepted: 21-12-2017 